\title{
Felicidade como Produto: Um Olhar Crítico sobre a Ciência da Psicologia Positiva
}

\author{
Caroline Tozzi Reppold ${ }^{1}$ \\ Universidade Federal de Ciências da Saúde de Porto Alegre, Porto Alegre-RS, Brasil \\ Daniela Sacramento Zanini, Daniela Cristina Campos, Margareth Regina G. Veríssimo de Faria \\ Pontifícia Universidade Católica de Goiás, Goiânia-GO, Brasil \\ Bruna Simões Tocchetto \\ Universidade Federal de Ciências da Saúde de Porto Alegre, Porto Alegre-RS, Brasil
}

\section{RESUMO}

A Psicologia Positiva é hoje uma ciência consolidada, e diversos são os achados que demonstram a eficácia de suas intervenções em prol da saúde e do bem-estar. Contudo, a expansão da PP em âmbito não acadêmico e a apropriação de seus ideais pela mídia indicam a necessidade de reflexão sobre a validade consequencial dos estudos na área. Nessa perspectiva, o artigo busca (a) fundamentar a PP e discutir sua expansão, considerando a incorporação dos preceitos da PP pela mídia e as consequências mercadológicas de uma concepção de felicidade autocentrada e utilitarista, alheia às demandas coletivas, (b) problematizar, em termos éticos e sociais, o uso de construtos positivos em práticas não acadêmicas e apresentar evidências de que intervenções envolvendo construtos positivos podem ter efeitos desfavoráveis às pessoas ou à sociedade, quando descontextualizadas, e (c) considerar diferenças epistemológicas das três ondas que caracterizam o movimento e discutir o futuro da área.

Palavras-chave: psicologia positiva; felicidade; intervenção psicológica; avaliação da eficácia terapêutica; validade consequencial.

\section{ABSTRACT - Happiness as a Product: A Critical Look at the Science of Positive Psychology}

Positive Psychology is now a consolidated science and there is much evidence that demonstrates the effectiveness of its interventions on health and well-being. However, the expansion of non-academic PP and the appropriation of its ideals by the media indicate the need for reflection on the consequential validity of studies in the area. From this perspective, the article aims to (a) characterize PP and discuss its expansion, considering the incorporation of precepts of PP by the media and the marketing consequences of a selfcentered and utilitarian conception of happiness, oblivious to collective demands, (b) problematize, in ethical and social terms, the use of positive constructs in non-academic practices and provide evidence that interventions involving positive constructs can have unfavorable effects on people or society when decontextualized, and (c) consider epistemological differences of the three waves that characterize the movement and discuss the future of the area.

Keywords: positive psychology; happiness; psychological Intervention; evaluation of therapeutic effectiveness; consequential validity.

\section{RESUMEN - La Felicidad como Producto: Una Mirada Crítica a la Ciencia de la Psicología Positiva}

La psicología positiva es ahora una ciencia consolidada y son diversos los hallazgos que demuestran la efectividad de sus intervenciones a favor de la salud y el bienestar. Sin embargo, la expansión de la PP en el ámbito no académico y la apropiación de sus ideales por los medios de comunicación indican la necesidad de reflexionar sobre la validez resultante de los estudios en el área. Desde esta perspectiva, el artículo busca (a) fundamentar la PP y discutir su expansión, considerando la incorporación de los preceptos de la PP por los medios de comunicación y las consecuencias de la comercialización de una concepción de felicidad egocéntrica y utilitarista, ajena a las demandas colectivas, (b) problematizar, en términos éticos y sociales, el uso de constructos positivos en prácticas no académicas y proporcionar evidencias de que las intervenciones que involucran construcciones positivas pueden tener efectos desfavorables en las personas o la sociedad cuando se descontextualizan, y (c) considerar las diferencias epistemológicas de las tres ondas que caracterizan el movimiento y discutir el futuro del área.

Palabras clave: psicología positiva; felicidad; intervención psicológica; evaluación de la eficacia terapéutica; validez consecuencial.

A Psicologia Positiva (PP) surge no início do último século como uma proposta de resgatar à ciência psicológica o interesse pelo estudo de atributos que contribuem para que as pessoas tenham uma vida mais saudável, feliz e realizada. Nesse sentido, a PP facilmente ganhou destaque no século XXI ao valorizar uma visão mais

${ }^{1}$ Endereço para correspondência: Laboratório de Pesquisa em Avaliação Psicológica. Rua Sarmento Leite, 245, 90050-170, Porto Alegre, RS. Tel.: (51) 3303-8826. E-mail: carolinereppold@yahoo.com.br Apoio institucional: CNPq e FAPERGS. 
holística das características humanas e ao centrar seus estudos na busca de evidências científicas (sobretudo por meio de ensaios clínicos controlados) que explicassem construtos, muitas vezes, relegados pela ciência psicológica, em razão da dificuldade de defini-los e mensurá-los (Seligman, 2019; Seligman \& Csikszentmihalyi, 2000; Snyder \& Lopez, 2002). Entre eles, o bem-estar e a felicidade, o amor, o otimismo, a gratidão e a espiritualidade.

Fato é que a Psicologia Positiva foi impulsionada pelo momento histórico da sua criação. $\mathrm{Na}$ virada do século, havia uma maior abertura das pessoas à reflexão sobre o que aspiravam para si e para o mundo e uma preocupação crescente com a busca de qualidade de vida fomentada pelos índices ascendentes de adoecimento mental observados em quase todos os países ocidentais. Também a consolidação de novas mídias sociais contribuiu para expansão da PP, à medida que essas inovações da época delineavam aos usuários novas formas de expor sentimentos, bens e valores, que resultaram em uma cultura de supervalorização de afetos positivos, nem sempre reais. Efetivamente, a Psicologia Positiva difundiu a discussão sobre o bem-estar e as potencialidades humanas entre diferentes públicos e diversas mídias, ganhando destaque no século XXI.

O crescente movimento da Psicologia Positiva no Brasil e no mundo pode ser observado por meio do aumento no número de publicações, pesquisas, cursos e especializações relacionados ao tema. Apesar disso, a expansão da PP e a apropriação de suas ideias pelo mercado e pela mídia têm indicado a necessidade de ponderações sobre a validade consequencial dos estudos na área, considerando que a PP não é uma panaceia em prol da promoção de saúde e felicidade e que as difusões de seus conceitos e intervenções podem ter efeitos adversos sobre as pessoas e a sociedade.

O presente artigo tem por objetivo apresentar a mudança de paradigma científico introduzida pela Psicologia Positiva ao longo de três momentos distintos (três ondas), discutindo as consequências sociais e éticas de sua expansão. Em específico, o artigo busca: (a) fundamentar a área e discutir sua expansão, (b) discutir a incorporação de preceitos da PP por parte da mídia e suas consequências mercadológicas, (c) problematizar, em termos éticos e sociais, o uso de construtos positivos em práticas não acadêmicas e alheias às evidências científicas, (d) apresentar evidências que demonstrem que práticas relacionadas a construtos positivos não são inócuas e, por vezes, podem ter efeitos adversos às pessoas ou à sociedade, quando realizadas de forma descontextualizada, e (e) discutir o desenvolvimento da PP como ciência, considerando as diferenças epistemológicas das três ondas que caracterizam o movimento e o futuro da área.

\section{Psicologia Positiva: Uma Mudança de Paradigma}

No artigo que demarca a criação da área, Seligman e Csikszentmihalyi (2000) destacam que o período Pós
II Guerra Mundial marcou o campo dos estudos na área de saúde mental pelo interesse quase exclusivo em diagnosticar sintomas e investigar estratégias que minimizassem o sofrimento e os efeitos da psicopatologia na vida cotidiana. Dessa feita, intenções iniciais da Psicologia no século XIX, como identificar talentos ou forças humanas e promover recursos psicossociais que fizessem com que as pessoas tivessem uma vida mais próspera, foram deixadas de lado em prol de interesses político-econômicos e da valorização de um modelo biomédico de compreensão do desenvolvimento humano, centrado em uma perspectiva individual.

Contudo, o modelo biomédico, com foco no tratamento e intervenções especializadas, mostrou-se ineficaz para os desafios da época. Nascia a compreensão de que a prevenção de problemas nem sempre é possível, mas a potencialização de recursos que possam contribuir para um desfecho saudável sim (Zanini, Faria, \& Campos, 2019).

A busca de evidências em prol de aspectos positivos do funcionamento humano em detrimento dos patologizantes não foi um esforço exclusivo da Psicologia, mas um movimento de diversas ciências, já observado em décadas anteriores (Marujo, Miguel Neto, \& Balancho, 2013). Nas ciências da saúde, a reconceituação de saúde proposta pela Organização Mundial de Saúde (OMS) décadas antes já era uma expressão disso. Até então definida como ausência de doenças, a saúde passou a ser definida pela OMS, a partir de 1948, como o completo bem-estar físico, mental e social, indicando um marco na compreensão do conceito e um incentivo ao estudo de aspectos positivos do funcionamento humano, ainda que essa definição tenha sido muito criticada por ser pouco operacional (Segre \& Ferraz, 1997).

Entre os psicólogos, um dos motivos que explica o súbito crescimento da área foi o interesse das pessoas em submeterem-se a intervenções psicológicas menos ortodoxas e mais breves, características de muitas intervenções positivas com evidências de eficácia ou efetividade (Bolier, Haverman, Westerhof, Smit, \& Bohlmeijer, 2013; Hutz \& Reppold, 2018; Reppold, Kaiser, \& Almeida, 2018; Reppold, Kaiser, D'azevedo, \& Almeida, 2018). Outro motivo é o fato de as técnicas baseadas em preceitos positivos aumentarem o senso de realização dos profissionais psicólogos que se propunham a desenvolver o hedonismo de seus clientes/pacientes.

Assim, preconizando o uso de métodos quantitativos e de delineamentos científicos rigorosos que primem pela busca de evidências científicas, sobretudo por meio de ensaios clínicos controlados (Seligman, Steen, Park, \& Peterson, 2005), a PP, desde sua criação, diferencia-se de alguns esforços anteriores. Sobretudo, por trazer à luz da ciência e à verificação empírica temas inerentes ao desenvolvimento humano e à autorrealização que antes eram considerados paralelos à investigação acadêmica, mas povoavam o interesse popular, por meio, por 
exemplo, de explanações não refutáveis, teorias não parcimoniosas ou de literatura de autoajuda. Embora alguns teóricos questionem se a PP poderia ser mesmo considerada um novo movimento científico, com mudanças de paradigmas vigentes, ou uma mobilização de atenção e de recursos financeiros para demandas (sociais e científicas) preteridas pela Psicologia Moderna (Diener, 2003; Duckworth, Steen, \& Seligman, 2005), não há dúvidas de que a origem da PP é acadêmica e que sua argumentação fomenta que as práticas associadas à busca do bem-estar e da autorrealização devam ser baseadas em evidências científicas.

\section{Problematizações acerca da Expansão da Psicologia Positiva}

A PP rapidamente expandiu-se no continente americano e encontrou eco entre profissionais de diversas formações vinculadas às áreas da saúde, da educação e das organizações (Diener, Pressman, Hunter, \& DelgadilloChase, 2017; Kim, Doiron, Warren, \& Donaldson, 2018; Macaskill, 2016; Machado, Gurgel, \& Reppold, 2017; Norrish, 2015). Assim, há de se ter cuidado para os riscos associados à disseminação da PP no estilo fast food, ou seja, para propagação da ideia de que a felicidade se reduz a um momento efêmero autocentrado ou a um produto que pode ser comprado ou consumido.

Ocorre que, embora a PP tenha origem acadêmica, o que se viu ao longo de sua expansão foi que o uso dado aos seus preceitos assumiu três caminhos distintos, que podem ser agrupados de acordo com o campo em que floresceram (acadêmico, paralelo à academia e na mídia).

1. No campo acadêmico, a expansão da área tomou o rumo da organização de estudos focados na busca de evidências que demonstrassem: (a) quais construtos são teoricamente consistentes pós investigação empírica e, portanto, representam um incremento em relação às teorias já existentes versus quais construtos são apenas sofismas (ex., Marujo et al., 2013), (b) quais instrumentos/técnicas são mais apropriados para avaliar a amplitude e especificidade do construto em questão (ex., Hutz, 2016), (c) quais intervenções são efetivas/eficazes versus quais são inócuas ou envolvem risco a seus participantes (ex., Bolier et al., 2013), (d) qual a validade consequencial dos avanços científicos encontrados e quais repercussões sócio-político-histórico-ideológico representam (ex., Diener, Heintzelman, Kushlev, Tay, Wirtz, Lutes, \& Oishi, 2017; Gruber et al., 2009).

2. Paralelo à academia, temas de interesse à Psicologia Positiva, como felicidade, bem-estar, gratidão, florescimento, resiliência, autorregulação e inteligência emocional, por exemplo, foram, por vezes, encapados em livros de autoajuda e práticas de desenvolvimento pessoal ou coaching. Embora essas publicações e práticas muitas vezes se utilizem de referências e conceitos científicos, observa-se que suas discussões geralmente são apresentadas de forma prescritiva e não há preocupação com o desenvolvimento de estudos que avaliem sua validade. Além disso, em alguns casos, são realizadas por pessoas sem formação, pouco interessadas em evidências científicas e/ou que desconsideram eventuais efeitos negativos das práticas ou a necessidade de contextualizá-las.

3. Na mídia, estudos desenvolvidos com base na Psicologia Positiva ganharam visibilidade. Contudo, algumas vezes foram usados de forma distorcida por campanhas de marketing, justificando ou legitimando um discurso de que a busca da felicidade é o maior objetivo da existência humana e um valor a ser alcançado. Nessa perspectiva, a felicidade é concebida, como uma condição fugaz, volátil e personificada. A ideia divulgada passa alheia a uma reflexão sobre os impactos sociais, políticos e ecológicos de se pensar que a felicidade pode ser atingida por comportamentos individualistas ou por bens de consumo. Em consequência, o bem-estar e as variáveis positivas que predizem uma vida saudável e harmoniosa são apresentados, em campanhas publicitárias e editoriais jornalísticos, como produtos a serem adquiridos/atingidos, explorados pelo marketing de forma utilitarista.

\section{Aspectos Mercadológicos da PP: A Incorporação de seu Discurso pela Mídia}

Atualmente, a busca por bem-estar e qualidade de vida aparece no discurso social como prioridade. Nessa perspectiva, frequentemente observa-se, na mídia, narrativas baseadas em jargões que apresentam a felicidade como uma necessidade intrínseca para o sucesso pessoal. Nessas explanações, a exaltação das sensações momentâneas de prazer regula uma forma de viver que busca constantemente alcançar essas sensações por meios de produtos, serviços, empregos, casa própria, filhos, ou outras metas de vida "padronizadas" no imaginário social (Brinkmann, 2017; Warren, 2009).

No aspecto mercadológico, a narrativa positiva é supervalorizada por meio de um sistema que se retroalimenta entre demandas e necessidades de consumo, estabelecendo-se como uma espiral em ascensão. Indivíduos querem comprar felicidade, e as empresas entendem que falar sobre sensações e experiências positivas e sobre qualidade de vida conectam os consumidores com suas marcas, tornando-as mais desejadas (Fogaça \& Perez, 2014). Um dos maiores clássicos publicitários que representam essa espiral é a Cola-Cola, que utiliza as construções de liberdade e felicidade em seu posicionamento de marca desde seus primórdios (Cassiano, 2008). No Brasil, seu primeiro comercial de televisão, em 1955, fala sobre a sensação de satisfação, prazer e entretenimento que a bebida traz por meio da chamada "Isto faz um bem". Em 2006, sua campanha era intitulada "Fábrica da Felicidade" (Tinelli \& Mazzei, 2014). Esses discursos publicitários demonstram o quanto sentimentos humanos 
vêm sendo fabricados sinteticamente e comercializados ao longo dos anos - sendo atualmente uma moeda de valor no mundo ocidental. Essa é uma realidade histórica e estreitamente imbricada à Psicologia, desde seus primórdios, com o trabalho de Watson. No entanto, o que se observa nos últimos anos é a apropriação, por parte da mídia, da legitimidade científica dos estudos sobre saúde e bem-estar para transformar produtos em meios de alcançar saúde, longevidade, plenitude, alegria etc. Essa expropriação do discurso científico, derivado de estudos de reconhecido mérito acadêmico, acaba por fazer com que os consumidores antes alcançados pelas campanhas de apelo emocional, agora sejam também capturados pela racionalidade.

Os autores Illouz (2007), Warren (2009) e Hochschild (2012) defendem que essas demandas surgem a partir do que eles intitulam de capitalismo emocional, no qual as transações econômicas e as emoções se influenciam simbioticamente. A partir dessa tese, os indivíduos agem como atores emocionais, motivados pelo imperalismo do discurso positivo, buscando trabalhar suas emoções com o objetivo de obter ganhos pessoais, direcionados a metas profissionais e/ ou financeiras. Como consequência, constrói-se uma cultura que valoriza as emoções e o autoconhecimento a partir do viés da vulnerabilidade, tratando sintomas depressivos ou ansiogênicos como gatilhos para o desenvolvimento pessoal/profissional. Dessa forma, os indivíduos são incentivados a buscarem soluções para deficits ora tratados como problemas pessoais, ao mesmo tempo estar em constante aperfeiçoamento em busca do bem-estar, da realização e da qualidade de vida (Warren, 2009; Brinkmann, 2017). Nesse discurso, o caráter dialógico dessas condições poucas vezes é considerado.

Diante desse cenário, a Psicologia Positiva torna-se atraente e palatável para o grande público por abordar temas que conversam com demandas atuais da sociedade; assim, vem sendo utilizada em diferentes contextos e aplicações, como na educação, em organizações e no desenvolvimento pessoal. Em contrapartida, autores criticam a Psicologia Positiva pela forma como ela pode potencializar a tirania do pensamento positivo, pois entendem que esse discurso acaba por supervalorizar contrutos relacionados ao bem-estar e rejeitar os deficits ou aspectos negativos da saúde mental (Held, 2002; Warren, 2009; Brinkmann, 2017). Logo, torna-se importante diferenciar o jargão positivo e a ciência.

De fato, alguns estudos mostram evidências que fundamentam o questionamento da promoção de variáveis positivas em determinados contextos e demonstram, inclusive, que em algumas situações promovê-las pode ser prejudicial. Esses resultados são importantes na medida em que contribuem para uma reflexão sobre a validade consequencial das intervenções baseadas em preceitos da PP.

\section{A PP é sempre Positiva? Reflexões sobre Validade Consequencial de suas Práticas}

A literatura da área é pródiga em mostrar que intervenções baseadas em preceitos da PP são eficazes. Em geral, os resultados obtidos por meio de ensaios clínicos randomizados mostram que as práticas investigadas têm tamanho de efeito pequeno, mas duradouro nos estudos de segmento, sendo as práticas mais eficazes aquelas desenvolvidas de forma contextualizada (Boulier et al., 2013; Chakhssi, Kraiss, Sommers-Spijkerman, \& Bohlmeijer, 2018; Reppold et al., 2018b).

Apesar disso, outros estudos demonstram que intervenções em PP também podem ter consequências negativas ou serem contraindicadas para populações específicas, comprometendo a validade consequencial dessas intervenções. Evidências baseadas nas consequências da testagem referem-se à avaliação das implicações sociais, sejam elas intencionais ou não, do uso de determinada técnica, de modo que se possa verificar se sua utilização está de acordo com a finalidade para qual foi criada, se é efetivamente útil (se seus resultados ajudam a definir estratégias terapêuticas ou políticas públicas, por exemplo), ou ainda se seu uso, de alguma forma, fere princípios morais ou direitos humanos (American Educational Research Association, American Psychological Association, \& National Council on Measurement in Education, 2014; Messick, 1995). O conceito de validade consequencial, como visto, é originário da avaliação de testes psicológicos, mas aqui propomos sua extensão à avaliação de técnicas de intervenção também. De acordo com Primi, Muniz e Nunes (2009), esse tipo de evidência não acrescenta informação propriamente sobre validade técnica, mas sobre as interpretações dos resultados de sua aplicação em contextos específicos e sobre o uso que é feito desses, indicando eventuais benefícios ou malefícios decorrentes aos indivíduos ou à sociedade. Nesse sentido, ocorre que achados científicos já indicam que as práticas envolvendo promoção de afetos, gratidão ou autoestima, por exemplo, não são inócuas e, por vezes, contradizem a crença popular de que "mal não faz".

Esse é o caso de algumas técnicas baseadas em mindfulness, amplamente difundidas na atualidade, são contraindicadas em determinados quadros de transtornos mentais pelo seu potencial em aumentar a sensação de desconforto físico e/ou emocional ou de agravar/precipitar sintomas psicológicos (como estresse ou desorientação), psicopatológicos (como ansiedade, delírios ou alucinações) e fisiológicos (como dor, inapetência, disfunções sensoriais ou crises de epilepsia) (Cebolla, Demarzo, Martins, Soler, \& Garcia-Campayo 2017).

Outros estudos mostram que intervenções focadas em aumentar a intensidade de emoções positivas podem ser prejudiciais a pessoas com um perfil combinado de impulsividade e busca de sensações (sensation seeking) (Herman, Critchley, \& Duka, 2018) e também àquelas que apresentam mania (Gruber et al., 2009), 
distúrbios alimentares (Cardi, Leppanen, \& Treasure, 2015; Evers, Dingemans, Junghans, \& Boevé, 2018) ou abuso de substâncias (Franken \& Muris, 2006; Krentzman, 2013), aumentando a exposição aos riscos que essas condições acarretam.

Para além dos contextos envolvendo quadros psicopatológicos, em população geral, intervenções focadas no aumento de afetos positivos também podem apresentar contraindicações. Pressman e Cohen (2005), em um estudo de revisão da literatura, reportaram evidências de que populações com altos índices de afetos positivos apresentam menos sintomas e dores físicas e maior longevidade. Contudo, indicam também que escores excessivamente altos de bem-estar estão associados a maior morbidade e mortalidade. Os autores discutem esse dado à luz da tendência desse grupo subestimar riscos e cuidados com a saúde.

Além disso, a revisão Pressman e Cohen (2005) mostra que a literatura é inconsistente sobre a relação entre afetos positivos e saúde. Alguns achados mostram que indução experimental de afetos positivos desencadeia uma excitação fisiológica com efeitos potencialmente prejudiciais na função imunológica, cardiovascular e pulmonar de indivíduos doentes.

Efeitos adversos também são relatados na literatura em relação a técnicas de promoção de gratidão em casos específicos. Embora intervenções para o desenvolvimento de gratidão estejam empiricamente associadas a benefícios para a saúde e bem-estar na população não clínica (ver Cunha, Pellanda, \& Reppold, 2018), evidências mostram que, entre alcoolistas, exercícios de gratidão são contraindicados àqueles que têm a abstinência como meta (Krentzman, 2017).

O estudo de Santos e Moré (2011) mostrou também que aumentar a gratidão entre pessoas submetidas a eventos de violência e assédio pode potencializar os riscos a que elas estão expostas. Os achados obtidos junto a mulheres que prestaram queixa policial de violência doméstica indicaram que eram altos os índices de insegurança e baixa autoestima dessas mulheres, e que, mesmo diante de situações de violência psicológica, humilhações, xingamentos, depreciação do seu modo de vestir e desqualificação de seu corpo, elas relatavam ser gratas pelo "cuidado" que recebiam de seus companheiros.

O mesmo acontece em relação à capacidade de perdoar. Um estudo longitudinal realizado por McNulty (2011) com casais recém-casados revelou uma associação positiva entre os relatos dos cônjuges sobre suas tendências para expressar perdão aos seus parceiros e relatos de agressão impetrada por esses parceiros. Especificamente, os cônjuges mais complacentes relataram com maior frequência situações de agressão física e psicológica durante os primeiros quatro anos de casamento; por sua vez, os menos tolerantes ao abuso relataram que as situações de agressão sofridas diminuíram ao longo do tempo. Esses resultados permitem concluir que a capacidade de perdão não é a panaceia para uma vida feliz.

Nessa linha, a pesquisa publicada por Campos (2016) demonstrou ainda haver uma relação entre violência e autoeficácia. Os achados indicaram que, embora a autoeficácia possa ser um fator de proteção à saúde (Zamani-Alavijeh, Araban, Harandy, Bastami, \& Almasian, 2019; Williams \& Rhodes, 2016), em contexto específico de violência, pode se constituir em fator de risco. Especificamente, os resultados evidenciaram que os jovens adultos que apresentavam forte crença de autoeficácia relataram, mais frequentemente, situações de violência sexual e violência testemunhada. A autora interpretou esse dado considerando que se avaliar como eficaz para lidar com situações de violência levava os jovens a se precaverem menos, colocando-se em maior risco para situações de violência. Esse achado vem ao encontro de outro estudo desenvolvido por Zanini, Campos, Faria, e Peixoto (2018), o qual investigou os efeitos de uma intervenção baseada em PP para prevenção de violências interpessoais e autorrelatadas e para promoção de saúde em adolescentes. Os resultados indicaram aumento no escore de todas as medidas positivas e diminuição dos índices de violências após a intervenção, à exceção da autoeficácia para situações de violência. Esse dado corrobora a hipótese de que participantes com altos níveis de autoeficácia acabam expostos mais frequentemente a situações de risco de violência.

Outros estudos que demonstram que as intervenções positivas não são inócuas e, quando descontextualizadas, podem trazer malefícios à saúde referem-se às estratégias para aumentar o otimismo das pessoas. Por um lado, pesquisas mostram que pessoas otimistas têm melhor saúde física, são mais longevas e tendem a fazer avaliações mais positivas do futuro, acreditando que serão mais felizes adiante, o que as deixa mais felizes no presente (Rasmussen, Scheier, \& Greenhouse, 2009). Mostram também que os otimistas avaliam melhor seus companheiros e suas relações afetivas (Srivastava, McGonigal, Richards, Butler, \& Gross 2006). Por outro, estudos demonstram que o excesso de otimismo pode levar a uma estimativa minimizada dos riscos aos quais o indivíduo está exposto e à negligência a cuidados preventivos (Hanoch, Rolison, \& Freund, 2019), aumentando as chances de consequências negativas. No contexto de jogos de azar, por exemplo, pessoas otimistas são menos propensas a reduzir suas apostas após sucessivas derrotas, estando mais vulneráveis a situações de gambling (Gibson \& Sanbonmatsu, 2004).

Também em relação à autoestima, a clássica revisão de Baumeister, Campbell, Krueger e Vohs (2003) reúne uma série de estudos empíricos para discutir os benefícios e malefícios de uma alta autoestima. Os autores discutem que professores, pais e terapeutas concentram esforços no aumento da autoestima, partindo do pressuposto de que a alta autoestima terá como desfecho maior 
sucesso acadêmico e profissional e melhores indicadores de saúde e de relacionamento, mas as pesquisas mostram que esses desfechos nem sempre se confirmam ou têm uma relação direta. Embora muitos benefícios sejam encontrados (relacionados, principalmente, à vivência de sentimentos agradáveis, ao enfrentamento do estresse e à tomada de iniciativa), entre as consequências negativas, os autores listam que adolescentes com autoestima muito elevada são mais propensos à experimentação de drogas $\mathrm{e}$ à prática sexual precoce, bem como à prática de bullying, à falta de empatia, a atos de preconceitos/discriminação e à ocorrência de uma sensação de segurança irreal, que os tornariam menos capazes de uma autoavaliação adequada. Os autores concluíram não haver evidências de que o aumento da autoestima (por intervenções terapêuticas ou programas escolares) resulte em benefícios diretos. Por essa razão, afirmam que as evidências não apoiam os esforços generalizados de pais e professores em aumentar a autoestima de crianças e adolescentes na esperança de que ela promova melhores resultados sociais, emocionais ou acadêmicos, visto que o elogio indiscriminado pode promover o narcisismo e ter consequências indesejáveis.

Nessa linha, observa-se a necessidade de se avaliar com cuidado as consequências de práticas, por muitos, consideradas positivas ou "inócuas". Atualmente, diferentes técnicas de promoção de autoestima, por exemplo, passaram a ser desenvolvidas e ofertadas em oficinas por pessoas sem formação, mesclando um discurso de empoderamento pessoal e de pretensa cientificidade que não tem base em evidências e que ignora o caráter dialógico do "verdadeiro" empoderamento social.

Em conjunto, esses estudos demonstram que intervenções baseadas em preceitos de PP podem ser estratégias eficazes para que as pessoas encontrem uma vida mais próspera, feliz e saudável. No entanto, cabe ao profissional capacitar-se teórica e tecnicamente para utilizá-los de forma adequada, nos contextos apropriados e levando em consideração variáveis concorrentes que possam interferir nos desfechos desejados. $\mathrm{Na}$ Psicologia Positiva, como em outras ciências, não há como supor resultados ou fazer generalizações sem base empírica. A PP preconiza, desde sua fundação, uma ciência baseada em evidências. Nessa perspectiva, os resultados acumulados desde então demonstram que as intervenções contextualizadas (atentas às condições individuais, históricas e sociais) são as mais eficazes (Reppold et al., 2018b), e que, como demonstrado aqui, práticas consideradas positivas podem ter efeitos adversos em determinadas circunstâncias.

\section{A Evolução da Psicologia Positiva como Ciência: As Três Ondas da PP}

A discussão apresentada neste texto ilustra que a Psicologia Positiva, ora já considerada uma abordagem científica consolidada (Seligman, 2019), encontra-se em um momento propício para uma nova reflexão sobre seu objeto de estudo, seus métodos e as implicações éticas, sociais e ideológicas de suas práticas. De acordo com Seligman (2002), a Psicologia Positiva está fundamentada em três pilares que norteiam sua investigação:

- O estudo das emoções positivas: consideradas como variáveis subjetivas, definidas pelo que os sujeitos pensam e pelo que se sentem;

- O estudo dos traços positivos: que podem ser considerados como forças e virtudes humanas;

- O estudo das instituições positivas: contextos nos quais a vida em comunidade é observada e onde valores coletivos podem ser promovidos.

O desenvolvimento da área mostra que, ao longo de duas décadas, o escopo da PP foi ampliado, partindo do estudo teórico de variáveis relacionadas à promoção da felicidade em direção à proposição e avaliação de intervenções práticas que pudessem ser aplicadas a contextos diversos, como a clínica e a escola, aumentando a chance de desfechos considerados positivos nessas áreas. Entretanto, o estudo das emoções positivas segue sendo o principal pilar da PP (Diener et al., 2017; Seligman, 2019) e remonta a uma reflexão sobre o modo como a felicidade é compreendida e fomentada pela área. De fato, são diversos os modelos teóricos e avaliativos que podem ser adotados para o estudo da felicidade. Alguns partem de uma premissa individual e consideram a felicidade como uma meta pessoal a ser alcançada. Outros a consideram a partir de uma perspectiva relacional.

No campo da PP, as duas principais abordagens são os modelos hedonista e eudaimônico de felicidade. $\mathrm{O}$ primeiro, deriva de uma corrente filosófica que propunha que uma "boa vida" seria marcada pela experiência de maximização do prazer e minimização da dor, sendo a felicidade concebida como a totalidade dos momentos hedônicos vivenciados pela pessoa (Peterson, Park, \& Seligman, 2005). Baseado nessa concepção, centrada nas emoções positivas e na redução de afetos negativos, está o conceito de bem-estar subjetivo, uma medida que abrange uma avaliação global sobre a vida a partir de dois componentes: a satisfação de vida (dimensão cognitiva) e os afetos (dimensão emocional) (Diener, Lucas, \& Oishi, 2002). Por sua vez, o modelo eudaimônico parte da ideia de que a felicidade está relacionada a um significado de vida e aos sentimentos evocados quando a pessoa se move em direção à autorrealização e seus propósitos de vida. Nessa abordagem, está ancorado o conceito de bem-estar psicológico, o qual envolve, em uma perspectiva desenvolvimental, a superação dos desafios existenciais de vida, e a consideração das seguintes dimensões psicológicas: autoaceitação, relações positivas com os outros, autonomia, domínio do meio, propósito de vida, e crescimento pessoal (Keye, Shmotkin, \& Ryff, 2002).

De acordo com Neto e Araújo (2019, p. 190), ambos tratam de "felicidades não hierarquizáveis, mas 
interdependentes, por vezes sobrepostas e, ao que parece, com elementos de universalidade, tanto quanto circunstanciais. Ou seja, com aspectos comuns em múltiplas culturas, mas igualmente afetados pelo contexto, momento histórico (...). Todas essas felicidades nos sublinham a complexidade do conceito e desaconselham potenciais simplismos na sua abordagem". Assim, guardadas as considerações sobre a complexidade do tema e o caráter reducionista de um julgamento de valor sobre os modelos teóricos existentes, o que se observa com o desenvolvimento da área da Psicologia Positiva é que muitas das intervenções propostas sob os preceitos da PP foram elaboradas a partir de uma perspectiva hedônica e autocentrada, baseada na crença de que a felicidade é fruto de um projeto individual intencional, em detrimento do bem-estar comum e de uma ontologia relacional. Nesse sentido, a área da PP tem sofrido consistentes críticas ao desenvolver práticas voltadas para um público específico, com acesso à educação e hábil quanto ao pensamento abstrato, à metacognição e à capacidade de planejamento. As críticas destacam também que a felicidade não pode ser pensada como uma meta egocêntrica e que as intervenções da área deveriam ter mais atenção à conjuntura econômica, política e social do contexto de vida de cada indivíduo e às demandas coletivas (Neto \& Araújo, 2019; White, 2017).

As análises até aqui apresentadas organizaram o desenvolvimento da Psicologia Positiva a partir de três ondas. A primeira onda, já descrita, destacava, na virada do século XXI, a importância da consideração de construtos tidos como positivos no estudo do desenvolvimento humano e da autorrealização e a necessidade de que esses fossem investigados no âmbito acadêmico. Nessa perspectiva, Seligman e os pesquisadores que o acompanhavam inicialmente estavam interessados em compreender o que faz as pessoas serem felizes. Assim, centrando-se em estudos sobre os processos e condições que contribuem para felicidade e o florescimento, propunham um modelo mais holístico de compreensão do funcionamento humano.

A segunda onda da Psicologia Positiva (SOPP) nasce de uma crítica ao caráter maniqueísta de se pensar os conceitos psicológicos como positivos ou negativos. Cunhado pela americana Barbara Held (2004), o termo SOPP chama atenção para a natureza ambivalente e dialética do bem-estar e nos convida a compreender o funcionamento humano integrando o olhar negativo ao positivo. Held (2004) argumenta que a natureza dialética do bem-estar é revelada por três princípios: (a) Princípio da avaliação - que refere a dificuldade de classificar determinados fenômenos como positivos ou negativos, uma vez que dependem do contexto em que ocorrem; (b) Princípio da covalência - que refere o fato de que um mesmo fenômeno pode despertar emoções distintas e sobrepostas; (c) Princípio da complementaridade - que refere que o bem-estar deve ser entendido a partir de um modelo dialético, sendo o bem-estar e o mal-estar dimensões ortogonais do funcionamento humano e não parte de um contínuo. Nessa perspectiva, o bem-estar não se resume à ausência de mal-estar, sendo ambas as condições complementares dada a natureza dialética das emoções.

A terceira onda da Psicologia Positiva parte de uma crítica ao foco que a área deu até então, à experiência intrapsíquica e a enfase no modelo dualista e não complementar de emoções positivas x negativas. Embora Seligman (2002) tenha considerado o estudo das instituições positivas como um dos pilares da PP, e tenha defendido que a PP fosse ampliada como política pública na área da educação e da saúde (Adler \& Seligman, 2016; Seligman, 2019), o que se observa, na prática das intervenções realizadas é, muitas vezes, uma abordagem ainda autocentrada e não integradora das emoções (positivas x negativas).

Dessa feita, a terceira onda chama atenção para os princípios já elencados na segunda onda de avaliação, covalencia e complementaridade dos construtos positivos sobre as demais experiências humanas. Neste sentido, mesmos os contrutos positivos devem ser avaliados à luz dos contextos onde são aplicados, pois interagem com os demais aspectos da vida do indivíduo e comunidade. Contudo, a terceira onda avança nas discussões ao propor que o desenvolvimento acrítico de postulados positivos, quando aplicados sem uma análise da situação contextual e de suas consequências, pode ser prejudicial à vida do indivíduo ao potencializar a ansiedade social e a opressão individual derivada da ideia de que o "sofrimento é um fracasso pessoal" ou ainda da fantasia de que é possível a construção da felicidade autêntica de modo alheio aos condicionantes sociais a que se está submetido. De fato, há de se considerar as normas sociais, políticas e contexto social que podem potencializar a vivência de estresse e sofrimento e, dessa forma, o bem estar não pode ser considerado isolado das relações que se estabelece (Dirth \& Adams, 2019).

A terceira onda também destaca a importância das relações estabelecidas entre os indivíduos com seus pares, seus grupos, instituições e comunidades e para necessidade de ações pró-sociais com vistas ao bem comum, não apenas focadas no desenvolvimento pessoal. Nessa perspectiva, destacam-se conceitos como o self relacional, os bens relacionais não instrumentais, a construção de comunidades participativas, a reciprocidade, a gratuidade, as formas pacíficas de interação e de compartilhamento de bens e a sustentabilidade ambiental, todos considerados como caminhos para o bem comum. Mais do que isso, considerados como respostas ao capitalismo emocional e ao consequente distanciamento das relações de intimidade que as tendências individualistas de satisfação do ego impostas pela ideologia vigente (Neto \& Araújo, 2019).

Dessa forma, a terceira onda amplia o escopo da discussão sobre bem-estar, abrangendo três elementos: 
o mercado (especialmente no que se refere aos bens privados e à eficiência), o Estado (pelas políticas de distribuição de recursos e garantia de direitos comuns) e a sociedade civil (aqui incentivada na organização de vínculos sociais cooperativos e de uma cultura de paz). Nas palavras de Neto e Araújo (2019, p.193-194),

"em tempos de déficit de relacionalidade (...) não podemos nos limitar a maximizar o benefício próprio de curto prazo, tantas vezes sensorial e irrefletido. É excepcional que fiquemos mais mindful, ou mais focados (...) mas essa ação tem que se ligar a outras dimensões da vida que nos envolve. Precisamos pensar a ciência da PP como um instrumento que cultive as virtudes cívicas e nos desinstala do atual conformismo apático e consumista e nos ajuda a pensar mais longe e mais crítica sobre as felicidades que almejamos e a justiça social”.

\section{Considerações Finais}

O presente artigo objetivou apresentar a mudança de paradigma científico introduzida pela Psicologia Positiva, discutindo sua expansão e os contextos onde sua aplicação deve ser realizada com cautela. Dessa forma, o presente artigo apresentou as bases histórico-culturais que culminaram no surgimento da PP e os fundamentos filosóficos de suas discussões, voltadas para o estudo científico de atributos que contribuem para que as pessoas tenham uma vida mais saudável, feliz e realizada.

A mudança de foco dos aspectos negativos, ora privilegiados pela ciência, para os aspectos positivos, agora alvo de estudos da PP, quando na virada do século, ecoou em meio a uma sociedade com preocupações crescentes com a busca de qualidade de vida e aspirações de uma vida melhor. Nesse sentido, observou-se uma rápida expansão da PP entre as diversas áreas do conhecimento científico, mas também entre outros campos de interesses, destacados neste artigo como campos não acadêmicos e relacionados à mídia. A análise dessa expansão exarcebada, sem compromisso com seus efeitos, permite afirmar que o uso generalizado de técnicas envolvendo construtos positivos de forma descontextualizada e acrítica, sem estudos científicos, produz consequências que se estendem ao campo da ética.

De forma falaciosa, a divulgação dessas técnicas pela população em geral (em redes sociais, blogs ou em sites/livros de autoajuda), assim como sua implementação por profissionais despreparados são, muitas vezes, vistas por esses como práticas inócuas, ou regidas pela lógica de que "se bem não fizer, mal não fazem". Porém, a literatura científica desfaz esse engano mostrando, por meio de evidências empíricas, que algumas intervenções baseadas em preceitos da PP não se aplicam a todos e, em alguns casos, podem trazer desfechos iatrogênicos. Assim, o presente artigo apresenta diversos estudos científicos que demonstram que, em grupos específicos ou em determinados contextos, a potencialização de construtos positivos pode ter efeitos adversos para as pessoas e para construção de uma visão social coletiva, distante de uma preocupação autocentrada. Em conjunto, os dados evidenciam que a PP não é uma ciência inócua. Ao contrário, intervenções focadas em construtos positivos podem produzir mudanças significativas na vida das pessoas e fomentar um senso de coletividade em prol do bem-estar social. No entanto, dependem de estudos que demonstrem as evidências de sua validade, inclusive em termos de validade consequencial. Ou seja, de avaliação que explicitem as implicações sociais, sejam elas intencionais ou não, do uso de determinada técnica, de modo que se possa verificar se sua utilização está de acordo com a finalidade para qual foi criada, se a prática é efetivamente útil (se seus resultados ajudam a definir estratégias terapêuticas ou políticas públicas, por exemplo), ou ainda se seu uso, de alguma forma, fere princípios ético/morais, direitos humanos ou reitera um discurso autocentrado, preocupado com o bem-estar individual, em detrimento de seus pares.

Por fim, o artigo apresenta o estado atual da arte dos estudos em PP, reunindo-os no que se denominou três ondas da PP, e aponta para o fato de que, na perspectiva atual, a felicidade proposta pela PP não pode se constituir como uma meta egocêntrica, fruto de um projeto individual, hedônico e autocentrado. Ao contrário, os estudos da PP filiados ao que se identifica como terceira onda postulam que a promoção da felicidade deve, necessariamente, estar ligada ao desenvolvimento do bem-estar comum e de uma ontologia relacional, que considera a conjuntura econômica, política e social do contexto de vida de cada indivíduo e as demandas coletivas (Neto \& Araújo, 2019; White, 2017). Apenas dessa forma, promover-se-á uma felicidade autêntica.

\section{Referências}

American Educational Research Association, American Psychological Association, \& National Council on Measurement in Education. (2014). Standards for educational and psychological testing. Washington, DC: American Educational Research Association.

Adler, A., \& Seligman, M. E. P. (2016). Using wellbeing for public policy: Theory, measurement, and recommendations. International Journal of Wellbeing, 6(1), 1-35. doi: 10.5502/ijw.v6i1.1 
Baumeister, R. F., Campbell, J. D., Krueger, J. I., \& Vohs, K. D. (2003). Does high self-esteem cause better performance, interpersonal success, happiness, or healthier lifestyles? Psychological Science in the Public Interest, 4(1), 1-44. doi: 10.1111/1529-1006.01431

Bolier, L., Haverman, M., Westerhof, G. J., Riper, H., Smit, F., \& Bohlmeijer, E. (2013). Positive Psychology interventions: A meta-analysis of randomized controlled studies. BMC Public Health, 13(1), 119. doi:10.1186/1471-2458-13-119

Brinkmann, S. (2017). Stand Firm: Resisting the self-improvement craze. UK: Politu Press.

Campos, D. C. (2016). Vitimização e Revitimização em Estudantes Universitários: influência de fatores individuais e sociais. (Tese de Doutorado não publicada). Pontifícia Universidade Católica de Goiás, Goiânia.

Cardi, V., Leppanen, J., \& Treasure, J. (2015). The effects of negative and positive mood induction on eating behaviour: A meta-analysis of laboratory studies in the healthy population and eating and weight disorders. Neuroscience Biobehavioral Reviews, 57, 299-309. doi: 10.1016/j.neubiorev.2015.08.011

Cassiano, C. M. (2008). Gerenciamento de marketing global: O caso Coca-Cola. Revista de Ciências Gerenciais, 12(15) 159-176. doi: $10.17921 / 1415-6571.2008 v 12 n 15 p 159-176$

Cebolla, A., Demarzo, M., Martins, P., Soler, J., \& Garcia-Campayo, J. (2017). Unwanted effects: Is there a negative side of meditation? A multicentre survey. Plos One, 12(9), e0183137. doi: 10.1371/journal.pone.0183137

Chakhssi, F., Kraiss, J. T., Sommers-Spijkerman, M., \& Bohlmeijer, E. T. (2018). The effect of positive psychology interventions on wellbeing and distress in clinical samples with psychiatric or somatic disorders: A systematic review and meta-analysis. BMC Psychiatry, 18(1), 211. doi:10.1186/s12888-018-1739-2

Cunha, L. F., Pellanda, L. C., \& Reppold, C. T. (2018). Intervenções em gratidão. Em C. S. Hutz \& C. T. Reppold (Eds.), Intervenções em Psicologia Positiva aplicadas à saúde (pp. 81-98). São Paulo: Leader.

Diener, E. (2003). What is positive about positive psychology: The curmudgeon and Pollyanna. Psychological Inquiry, 14(2), 115-120. doi:10.1207/S15327965PLI1402_03

Diener, E., Heintzelman, S. J., Kushlev, K., Tay, L., Wirtz, D., Lutes, L. D., \& Oishi, S. (2017). Findings all psychologists should know from the new science on subjective well-being. Canadian Psychology, 58(2), 87-104. doi: 10.1037/cap0000063

Diener, E., Lucas, R., \& Oishi, S. (2002). Subjective well-being: The science of happiness and life satisfaction. Em C. Snyder \& S. Lopez (Eds.). Handbook of Positive Psychology (pp. 63-73). New York: Oxford University Press.

Diener, E., Pressman, S., Hunter, J., \& Delgadillo-Chase, D. (2017). If, why, and when subjective well-being influences health, and future needed research. Applied Psychology, Health and Well-Being, 9(2), 133-67. doi: 10.1111/aphw.12090.

Dirth, T. P., \& Adams, G. A. (2019). Decolonial theory and disability studies: On the modernity/coloniality of ability. Journal of Social and Political Psychology, 7(1), 260-289. doi:10.5964/jspp.v7i1.762

Duckworth, A. L., Steen, T. A., \& Seligman, M. E. P. (2005). Positive psychology in clinical practice. Annual Reviews of Clinical Psychology, 1 , 629-651. doi:10.1146/annurev.clinpsy.1.102803.144154

Evers, C., Dingemans, A., Junghans, A.F., \& Boevé, A. (2018). Feeling bad or feeling good, does emotion affect your consumption of food? A meta-analysis of the experimental evidence. Neuroscience \& Biobehavioral Reviews, 92, 195-208. doi: 10.1016/j.neubiorev.2018.05.028

Fogaça, J., \& Perez, C. (2014). Felicidade adjetivada: Polifonia conceitual, imperativo social. Intercom - RBCC, 37(1), 217-241. doi: 10.1590/ rbcc.v37i1.1890

Franken, I. H., \& Muris, P. (2006). BIS/BAS personality characteristics and college students' substance use. Personality and Individual Differences, 40(7), 1497-1503. doi:10.1016/j.paid.2005.12.005

Gibson, B., \& Sanbonmatsu, D. M. (2004). Optimism, pessimism, and gambling: The downside of optimism. Personality and Social Psychology Bulletin, 30(2), 149-160. doi: 10.1177/0146167203259929

Gruber, J., Culver, J. L., Johnson, S. L., Nam, J. Y., Keller, K. L., \& Ketter, T. A. (2009). Do positive emotions predict symptomatic change in bipolar disorder? Bipolar Disorders, 11(3), 330-336. doi:10.1111/j.1399-5618.2009.00679.x

Hanoch, Y., Rolison, J., \& Freund, A. M. (2019). Reaping the benefits and avoiding the risks: Unrealistic optimism in the health domain. Risk Analysis, 39(4), 792-804. doi:10.1111/risa.13204

Held, B. S. (2002). The tyranny of the positive attitude in America: Observation and speculation. Journal of Clinical Psychology, 58(9), 965-992. doi: 10.1002/jclp.10093

Held, B. (2004). The negative side of Positive Psychology. Journal of Humanistic Psychology, 44(1), 9-46. doi: 10.1177/0022167803259645

Herman, A. M., Critchley, H. D., \& Duka, T. (2018). Risk-taking and impulsivity: The role of mood states and interoception. Frontiers in Psychology, 9, 1625. doi:10.3389/fpsyg.2018.01625

Hochschild, A. R. (2012). Managed heart: Commercialization of human feeling. California: University of California Press.

Hutz, C. S. (2016). Avaliação em Psicologia Positiva: Técnicas e medidas. São Paulo: CETEPP Hogrefe.

Hutz, C. S., \& Reppold, C. T. (2018). Intervenções em Psicologia Positiva na área da saúde. São Paulo: Leader.

Illouz, E. (2007). Cold intimacies: The making of emotional capitalism. Oxford and Malden, MA: Polity Press.

Keyes, C. L. M., Shmotkin, D., \& Ryff, C. D. (2002). Optimizing well-being: The empirical encounter of two traditions. Journal of Personality and Social Psychology, 82(6), 1007-1022. doi: 10.1037/0022-3514.82.6.1007

Kim, H., Doiron, K., Warren, M. A., \& Donaldson, S. I. (2018). The international landscape of Positive Psychology research: A systematic review. International Journal of Wellbeing, 8(1), 50-70. doi:10.5502/ijw.v8i1.651

Krentzman A. R. (2013). Review of the application of positive psychology to substance use, addiction, and recovery research. Psychology of Addictive Behaviors: Journal of the Society of Psychologists in Addictive Behaviors, 27(1), 151-165. doi:10.1037/a0029897

Krentzman, A. R. (2017). Gratitude, abstinence, and alcohol use disorders: Report of a preliminary finding. Journal of Substances Abuse Treatment, 78, 30-36. doi:10.1016/j.jsat.2017.04.013.

Macaskill, A. (2016). Review of Positive Psychology applications in clinical medical populations. Healthcare, 4(3), 66. doi:10.3390/ healthcare 4030066

Machado, F. A. Gurgel, L., \& Reppold, C. T. (2017). Intervenções em Psicologia Positiva na reabilitação de adultos e idosos: Revisão da literatura. Estudos de Psicologia (Campinas), 34(1), 119-130. doi:10.1590/1982-02752017000100012

Marujo, H., Miguel Neto, L., \& Balancho, L. S. F. (2013). Emergência, desenvolvimento e desafios da Psicologia Positiva: Da experiência subjetiva à mudança social. Estudos Contemporâneos de Subjetividade, 3(2), 180-201. 
Messick, S. (1995). Validity of psychological assessment: Validation of inferences from persons' responses and performances as scientific inquiry into score meaning. American Psychologist, 50(9), 741-749. doi: 10.1037/0003-066X.50.9.741

McNulty J. K. (2011). The dark side of forgiveness: The tendency to forgive predicts continued psychological and physical aggression in marriage. Personality \& Social Psychology Bulletin, 37(6), 770-783. doi:10.1177/0146167211407077

Neto, L. M., \& Marujo, H. Á. (2019). Felicidade para o bem comum: Das intervenções em Psicologia Positiva à educação para a paz global sustentável. Em C. Reppold \& L. S. Almeida. (Eds.). Psicologia Positiva: Educação, Saúde e Trabalho (pp. 189-210). Minho: CERPSI.

Norrish, M. (2015). Positive Education: The Geelong Grammar School Journey. Oxford: University Press.

Peterson, C., Park, N. \& Seligman, M.E. (2005). Orientations to happiness and life satisfaction: The full life versus the empty life. Journal of Happiness Studies, 6(1), 25-41.

Pressman, S. D., \& Cohen, S. (2005). Does Positive Affect Influence Health? Psychological Bulletin, 131(6), 925-971. doi: 10.1037/00332909.131.6.925

Primi, R., Muniz, M., \& Nunes, C.H.S.S. (2009). Definições contemporâneas de validade de testes psicológicos. Em C.S. Hutz (Eds.). Avanços e polêmicas em avaliação psicológica (pp. 243-265). São Paulo: Casa do Psicólogo.

Rasmussen, H. N., Scheier, M. F., \& Greenhouse, J. B. (2009). Optimism and physical health: A meta-analytic review. Annals of Behavioral Medicine: A Publication of the Society of Behavioral Medicine, 37(3), 239-256. doi:10.1007/s12160-009-9111-x

Reppold, C. T., Kaiser, V., \& Almeida, L. S. (2018a). Intervenções de Psicologia Positiva no contexto da Psicologia Escolar. Em T. Nakano. (Eds.). Psicologia Positiva aplicada à educação (pp.7-18). São Paulo: Vetor Editora.

Reppold, C. T., Kaiser, V., D'Azevedo, L., \& Almeida, L. (2018b). Intervenções em Psicologia Positiva na área da saúde: O que os ensaios clínicos informam sobre a efetividade dessas intervenções? Em C. Hutz \& C. T. Reppold. (Eds.). Intervenções em Psicologia Positiva aplicadas à saúde (pp. 11-42). São Paulo: Leader.

Santos, A. C., \& Moré, C. L. (2011). Impacto da violência no sistema familiar de mulheres vítimas de agressão. Psicologia: Ciência e Profissão, 31(2), 220-235. doi: 10.1590/S1414-98932011000200003

Segre, M., \& Ferraz, F. R. C. (1997). O conceito de saúde. Revista de Saúde Pública, 31(5), 538-542. doi:10.1590/s0034-89101997000600016

Seligman, M. E. P. (2002). Positive psychology, positive prevention and positive therapy. Em C. Snyder \& S. Lopez (Eds.). Handbook of Positive Psychology (pp.3-9). New York: Oxford.

Seligman, M. E. P. (2019). Positive Psychology: A personal history. Annual Review of Clinical Psychology, 15, 1-23. doi: 10.1146/annurevclinpsy-050718-095653

Seligman, M. E. P., \& Csikszentmihalyi, M. (2000). Positive Psychology: An introduction. American Psychologist, 55(1), 5-14.

Seligman, M. E. P., Steen, T. A., Park, N., \& Peterson, C. (2005). Positive Psychology progress: Empirical validation of interventions. American Psychologist, 60(5), 410-421. doi: 10.1037/0003-066X.60.5.410

Snyder, C. R., \& Lopez, S. J. (2002). Handbook of Positive Psychology. New York: Oxford.

Srivastava, S., McGonigal, K. M., Richards, J. M., Butler, E. A., \& Gross, J. J. (2006). Optimism in close relationships: How seeing things in a positive light makes them so. Personality Processes and Individual Differences, 91(1), 143-153. doi: 10.1037/0022-3514.91.1.143

Tinelli, B., \& Mazzei, V. (2014, maio). Redação publicitária: Análise das propagandas "Isto Faz Um Bem" e "Fábrica de Felicidade" da CocaCola. Resumo apresentado no XIX Congresso de Ciências da Comunicação na Região Sudeste, Vila Velha. Recuperado de http://www. portalintercom.org.br/anais/sudeste2014/resumos/R43-1162-1.pdf

Warren, S. (2009). What's wrong with being positive? Em N. Garcea, S. Harrington \& A. Linley (Eds.) Oxford Handbook of Positive Psychology and Work (pp. 313-322). New York: Oxford Library of Psychology.

White, S. C. (2017). Relational wellbeing: Re-centring the politics of happiness, policy and the self. Policy E Politics, 45(2), 121-36. doi: 10. $1332 / 030557317$ X14866576265970

Williams, D. M., \& Rhodes, R. E. (2016). The confounded self-efficacy construct: Conceptual analysis and recommendations for future research. Health Psychology Review, 10(2), 113-128. doi:10.1080/17437199.2014.941998

Zamani-Alavijeh, F., Araban, M., Harandy, T. F., Bastami, F., \& Almasian, M. (2019). Sources of health care providers' self-efficacy to deliver health education: A qualitative study. BMC Medical Education, 19(1), 16. doi:10.1186/s12909-018-1448-z

Zanini, D. S., Campos, D. C., Faria, M. R. G. V., \& Peixoto, E. M. (2018). Prevenção de violência por meio de intervenções positivas. Em C. S. Hutz \& C. T. Reppold (Eds.), Intervenções em Psicologia Positiva aplicadas à Saúde (pp. 201-236). São Paulo: Leader.

Zanini, D. S., Faria, M. R. G. V., \& Campos, D. C. (2019). Psicologia Positiva e Psicologia da Saúde: Uma articulação possível e necessária. Em C. T. Reppold \& L. S. Almeida (Eds.). Psicologia Positiva: Educação, Saúde e Trabalho (pp. 73-104). Minho: Cerpsi.

recebido em agosto de 2019 aprovado em outubro de 2019

\section{Sobre as autoras}

Caroline Tozzi Reppold é psicóloga, doutora em Psicologia pela UFRGS, com pós-doutorado em Psicologia pela UFRGS, em Avaliação Psicológica pela USF e em Ciências da Educação pela Universidade do Minho, professora associada na UFCSPA, atua na graduação em Psicologia e no pós-graduação (PPGs Psicologia e Saúde, Ciências da Saúde, e Ciências da Reabilitação) e bolsista produtividade do CNPq.

Daniela Sacramento Zanini é psicóloga, doutora em Psicologia pela Universidad de Barcelona (Espanha), com pós-doutorado pela mesma instituição, e professora adjunta II do curso de graduação e pós-graduação em Psicologia da Pontifícia Universidade Católica de Goiás (PUC Goiás).

Daniela Cristina Campos é psicóloga, doutora em Psicologia pela PUC Goiás e professora do curso de graduação em Psicologia da PUC Goiás.

Margareth Regina G. Veríssimo de Faria é psicóloga, doutora em Psicologia pela PUC Goiás e professora do curso de graduação e pós-graduação em Psicologia da PUC Goiás e UniEvangélica.

Bruna Simões Tocchetto é publicitária, especialista em Dinâmicas de Grupos pela SBDG e mestranda do PPG Psicologia e Saúde/ UFCSPA. Atualmente, trabalha com pesquisa e consultoria de estratégia e criatividade para empresas. 